\title{
Selective and augmented $\beta$-glucuronidase expression combined with DOX-GA3 application elicits the potent suppression of prostate cancer
}

\author{
LONGXIN WANG $^{1 *}$, JIE DONG $^{1 *}$, MING WEI $^{2^{*}}$, WEIHONG WEN ${ }^{3}$, \\ JIANPING GAO ${ }^{1}$, ZHENGYU ZHANG ${ }^{1}$ and WEIJUN QIN ${ }^{2}$ \\ ${ }^{1}$ Department of Urology, Jinling Hospital, School of Medicine, Nanjing University, Nanjing, Jiangsu 210002; \\ ${ }^{2}$ Department of Urology, Xijing Hospital, Fourth Military Medical University, Xi'an, Shaanxi 710032; ${ }^{3}$ State Key Laboratory \\ of Cancer Biology, Department of Immunology, Fourth Military Medical University, Xi'an, Shaanxi 710032, P.R. China
}

Received August 28, 2015; Accepted October 19, 2015

DOI: 10.3892/or.2015.4454

\begin{abstract}
The present study was carried out to evaluate the specific and amplified $\beta$-glucuronidase $(\beta \mathrm{G})$ expression in prostate cancer cells by using a prostate-specific antigen (PSA) promoter-controlled bicistronic adenovirus and to evaluate the specific killing of prostate cancer cells after the application of the prodrug DOX-GA3. Bicistronic adenoviral expression vectors were constructed, and the effectiveness of specific and amplified expression was evaluated using luciferase and EGFP as reporter genes. $\beta \mathrm{G}$ expression was detected in $\mathrm{LNCaP}$ cells after they were infected with the $\beta \mathrm{G}$-expressing PSA promoter-controlled bicistronic adenovirus. MTT assays were conducted to evaluate the cytoxicity on the infected cells after the application of the prodrug DOX-GA3. Tumor growth inhibition was also evaluated in nude mice after treatment with the $\beta$ G-expressing adenovirus and DOX-GA3. Selective and amplified expression was observed in the PSA-producing LNCaP cells, but not in the PSA-non-producing DU145 cells. Potent cytotoxity and a strong bystander effect were observed in the LNCaP cells after infection with the $\beta \mathrm{G}$-expressing adenovirus and the application of DOX-GA3. Intravenous injection of a GAL4 regulated bicistronic adenovirus vector constructed to express $\beta \mathrm{G}$ under the control of the PSA promoter (Ad/PSAP-GV16- $\beta$ G) and the application of
\end{abstract}

Correspondence to: Dr Zhengyu Zhang, Department of Urology, Jinling Hospital, School of Medicine, Nanjing University, 305 East Zhongshan Road, Nanjing, Jiangsu 210002, P.R. China

E-mail: zhangzy20150828@sina.com

Dr Weijun Qin, Department of Urology, Xijing Hospital, Fourth Military Medical University, 127 Changle West Road, Xi'an, Shaanxi 710032, P.R. China

E-mail: qinweij@fmmu.edu.cn

*Contributed equally

Key words: $\beta$-glucuronidase, DOX-GA3, GAL4-VP16 prostate cancer
DOX-GA3 strongly inhibited tumor growth and prolonged the survival time of tumor-bearing nude mice. Selective and amplified $\beta \mathrm{G}$ expression together with the prodrug DOX-GA3 had an increased antitumor effect, showing great potential for prostate cancer therapy.

\section{Introduction}

Prostate cancer is the most commonly diagnosed cancer and the second leading cause of cancer-related deaths among males (1). Although commonly used treatments (surgery, radiation therapy and chemotherapy) can reduce tumor burden and prolong survival time, prostate cancer is not curative in about a half of patients. Together with the high recurrence rate, there is a strong demand to develop more effective strategies for prostate cancer therapy (2-4). Gene therapy provides a promising treatment strategy for cancer therapy, among which suicide gene therapy is considered as one of the most effective ones.

$\beta$-glucuronidase $(\beta \mathrm{G})$ is a type of acid hydrolase present in the microsomes and lysosomes of many human tissues, which can convert nontoxic glucuronide prodrugs with glycoside bonds into toxic glucuronic acid (5). The most commonly used prodrug is $\mathrm{N}$-[4-doxorubicin- $\mathrm{N}$-carbonyl (oxymethyl) phenyl] $O$ - $\beta$-glucuronyl carbamate (DOX-GA3), which can be converted into toxic DOX by $\beta \mathrm{G}(6,7)$. After successful conversion, not only the cells that express $\beta \mathrm{G}$ but the neighboring $\beta$ G-non-expressing cells are effectively killed, showing a strong bystander effect, which is a typical phenomenon in suicide gene therapy $(8,9)$. $\beta \mathrm{G}$ together with its glucuronide prodrug has been considered as a new suicide gene therapy system $(5,10-12)$. In addition, as it is a human protein, $\beta \mathrm{G}$ can avoid the immunogenicity of other suicide genes such as thymidine kinase (TK) and cytosine deaminase (CD), thus showing greater potential for clinical application.

For successful application of suicide gene therapy, specific high expression of the suicide gene in cancer cells is of vital importance for the specific killing of cancer cells. In some larger tumors, such as pancreatic carcinoma and ovarian carcinoma, $\beta \mathrm{G}$ is overexpressed and high levels of $\beta \mathrm{G}$ are present in the necrotic area, so a directly applied glucuronide prodrug 
can be converted into a toxic drug in these tumors and specifically kill the cancer cells $(7,13,14)$. But actually, most other tumors may not have increased $\beta \mathrm{G}$ expression or do not have necrosis, thus monotherapy by using a glucuronide prodrug of $\beta G$ is inapplicable. Under such conditions, targeted delivery or high expression levels of a suicide gene is the key factor for successful therapy.

For the targeted killing of cancer cells, selective expression of $\beta \mathrm{G}$ is of vital importance. The prostate-specific antigen (PSA) is known to be highly specific in prostate tissues, and has been used for targeted expression of therapeutic genes in prostate cancer. However, the relatively low transcription efficiency has limited its further application $(15,16)$. In the present study, a GAL4-regulated bicistronic adenoviral vector (Ad/PSAP-GV16- $\beta$ G) was constructed to express $\beta G$ under the control of the PSA promoter. In such a vector, the PSA promoter controls the expression of GAL4/VP16, which can transactivate the $\beta \mathrm{G}$ expression controlled by the GAL4/TATA promoter. This expression system was used to evaluate whether this $\beta \mathrm{G} / \mathrm{DOX}-\mathrm{GA} 3$ suicide gene system may have application prospect for the therapy of prostate cancer.

\section{Materials and methods}

The protocol of the present study was approved by the Ethics Committee of Jinling Hospital, in compliance with the Helsinki Declaration.

Cell lines. LNCaP, which is a prostate cancer cell line that expresses PSA, and DU145, which is a prostate cancer cell line from brain metastasis that does not express PSA, were cultured in RPMI-1640 media supplemented with $10 \%$ fetal bovine serum. Cells were maintained at $37^{\circ} \mathrm{C}$ in a humidified incubator with $5 \% \mathrm{CO}_{2}$.

Genomic DNA extraction, cDNA isolation and PCR amplification. The primers used to amplify the predicted PSA promoter were forward, 5'-tttagatcttagaggatctgtggaccacaa-3' and reverse, 5'-tttaagcttggtgacacagctctccgggtg-3'. PCR was performed using Ex Taq HS (Takara, Shiga, Japan) with the following program: $95^{\circ} \mathrm{C}$ for $2 \mathrm{~min}, 30$ cycles of $95^{\circ} \mathrm{C}$ for $30 \mathrm{sec}, 55^{\circ} \mathrm{C}$ for $30 \mathrm{sec}$ and $72^{\circ} \mathrm{C}$ for $30 \mathrm{sec}$, and a final step of $7 \mathrm{~min}$ at $72^{\circ} \mathrm{C}$.

Total RNA was harvested from cultured HepG2 cells using the RNeasy Mini kit (Qiagen, Hilden, Germany). Total RNA $(1 \mu \mathrm{g})$ was reverse-transcribed into $20 \mu \mathrm{l}$ cDNA using the PrimeScript PCR kit (Takara). The primers used to amplify the $\beta \mathrm{G}$ transcript were forward, 5'-tttgagctcatggccegggggtcggcggtt gcc-3' and reverse, 5'-aaacctaggagttcatttgcccgacaaaaggttt-3'. PCR was performed with the following program: $95^{\circ} \mathrm{C}$ for $2 \mathrm{~min}, 30$ cycles of $95^{\circ} \mathrm{C}$ for $30 \mathrm{sec}, 55^{\circ} \mathrm{C}$ for $30 \mathrm{sec}$ and $72^{\circ} \mathrm{C}$ for $30 \mathrm{sec}$, and a final step of $7 \mathrm{~min}$ at $72^{\circ} \mathrm{C}$. PCR products were analyzed using agarose gel electrophoresis and cloned into the pcDNA3.1 (Takara) vector for sequencing to ensure that the sequence was correct.

Plasmid construction and preparation of the bicistronic adenoviruses. The DNA fragments encoding the human PSA promoter (PSAP) and $\beta \mathrm{G}$ were amplified and subcloned. The pshuttle-GT-TRAIL and the pshuttle-hTERT-GV16-TRAIL plasmid were kindly provided by Dr B. Fang (17). The new bicistronic shuttle vector pshuttle-PSAP-GV16- $\beta$ G (Fig. 1) was constructed as follows. i) The TRAIL gene in the shuttle plasmid pshuttle-hTERT-GV16-TRAIL was replaced by the $\beta G$ gene to obtain the pshuttle-hTERT-GV16- $\beta$ G plasmid and ii) the hTERT promoter in pshuttle-hTERT-GV16- $\beta$ G was replaced by PSAP to obtain the pshuttle-PSAP-GV16- $\beta \mathrm{G}$ plasmid. The shuttle vector pshuttle-PSAP- $\beta$ G was constructed by replacing the GT promoter and the TRAIL gene in pshuttle-GT-TRAIL with PSAP and the $\beta$ G gene.

Adenoviral vectors were constructed as described previously (12). Briefly, the shuttle plasmids were cotransfected into 293 cells along with a 35-kb ClaI fragment from adenovirus type 5 . Then, recombinant vector Ad/PSAP- $\beta \mathrm{G}$ and Ad/PSAP-GV16- $\beta$ G were generated by homologous recombination and plaque-purified. The expression cassette sequence was then confirmed by DNA sequencing. The adenoviral vectors Ad/PSAP-GV16-luc, Ad/PSAP-GV16-EGFP, Ad/PSAP-Luc and Ad/PSAP-EGFP were also constructed in a similar manner. Virus titers were determined by optical absorbance at $\mathrm{A}_{260 \mathrm{~nm}}$ (one $\mathrm{A}_{260 \mathrm{~nm}}$ unit $=10^{12}$ particles $/ \mathrm{ml}$ ) and by plaque assay. Titers determined by $\mathrm{A}_{260}$ (i.e., viral particles) were used in all of the experiments. Particle: plaque ratios normally fell between 30:1 and 100:1. All of the viral preparations were free of contamination by E11 adenovirus and endotoxin.

Western blot analysis. Protein extracts were prepared from cultured cells or tumor tissue after they were homogenized in lysis buffer $(50 \mathrm{mM} \mathrm{NaCl}, 50 \mathrm{mM}$ EDTA, $1 \%$ Triton $\mathrm{X}-100)$ containing protease inhibitor cocktail (Roche, Indianapolis, IN, USA). Cell lysates (30 $\mu \mathrm{g})$ were separated on $12 \%$ sodium dodecyl sulfate polyacrylamide gel electrophoresis (SDS-PAGE) and transferred onto polyvinylidene difluoride membranes (Amersham Pharmacia Biotech, Uppsala, Sweden) according to standard protocol. The membranes were then blocked in phosphate-buffered saline (PBS) containing 5\% bovine serum albumin (BSA) and $0.05 \%$ Tween-20 for $2 \mathrm{~h}$ at room temperature, before being incubated with the appropriate primary antibody [anti-human $\beta$ G (1:1,000; Molecular Probes Life Technologies, Carlsbad, CA, USA) or anti- $\beta$-actin (1:2,500; Santa Cruz Biotechnology, Santa Cruz, CA, USA)] overnight at $4^{\circ} \mathrm{C}$. The membranes were washed with PBS containing $0.05 \%$ Tween and then incubated with the appropriate secondary antibody $(1: 10,000)$ for $1 \mathrm{~h}$ at room temperature. The bands were visualized using MultiImage $^{\mathrm{TM}}$ Light Cabinet Filter Positions (Alpha Innotech, San Leandro, CA, USA).

MTT assay. For the 3-(4,5-dimethylthiazol-2-yl)-2,5-diphenyltetrazolium bromide (MTT) assay, the LNCaP and DU145 cells were plated in 96-well plates at a density of 1,000 cells/well before infection with the control adenovirus, Ad/PSAP- $\beta \mathrm{G}$ or Ad/PSAP-GV16- $\beta$ G. At $48 \mathrm{~h}$ after the infection, different concentrations of DOX-GA3 or $10 \mu \mathrm{g} / \mathrm{ml}$ DOX-GA3 was added and incubated for the indicated times. Aliquots of $20 \mu \mathrm{l}$ of $5 \mathrm{mg} / \mathrm{ml}$ MTT (Sigma, St Louis, MO, USA) in PBS were added to each well, and the cells were incubated for another $4 \mathrm{~h}$ followed by the addition of $150 \mu 1$ dimethyl sufoxide (DMSO). The $A_{570 \mathrm{~nm}}$ values were assayed in a Sunrise microplate reader 
(Tecan, Groedig, Austria). The mean and standard deviations of three parallel samples were calculated.

Tumor growth model. BALB/c nude mice (4 to 6-weeks old, body weight 20-30 g) were purchased from National Rodent Laboratory Animal Resources, Shanghai Branch (Shanghai, China) and maintained under specific pathogen-free condition. The experiment was approved by the Animal Use and Care Committee of Jinling Hospital, School of Medicine, Nanjing University. The mice were inoculated subcutaneously (s.c.) with $2 \times 10^{6}$ human PSA-producing prostate cancer LNCaP cells. Tumors were allowed to grow until they reached a diameter of $0.3-0.5 \mathrm{~cm}$ (day 0 ). The mice were then randomly divided into different treatment groups. Six mice were included in each group.

In vivo treatment with the recombinant bicistronic adenovirus. To detect the selective expression of luciferase or EGFP in vivo, $1 \times 10^{8} \mathrm{PFU}$ of the control adenovirus, Ad/PSA-Luc or Ad/PSAP-EGFP were injected into the mice bearing the LNCaP tumors by tail vein. Mice were sacrificed $48 \mathrm{~h}$ after the infection and tissues were lysed for luciferase activity detection or snap-frozen for cryosectioning for the observation of EGFP by fluorescence microscopy.

To detect the antitumor effect of targeted $\beta \mathrm{G}$ expression plus prodrug DOX-GA3 in vivo, $1 \times 10^{8} \mathrm{PFU}$ of the control adenovirus, Ad/PSAP-GV16- $\beta$ G or Ad/PSAP- $\beta$ G was administered by an intravenous injection into the lateral tail, and $500 \mathrm{mg} / \mathrm{kg}$ DOX-GA3 was simultaneously injected. The maximum tolerated dose (MTD) of DOX-GA3 $(500 \mathrm{mg} / \mathrm{kg}$ weekly $\mathrm{x} 2$ ) was determined according to a previous report (6). The treatment was performed twice, once a week. The tumor sizes were determined by measuring two diameters perpendicular to each other with a caliper every 3 days. The tumor volume (V) was estimated using the equation $\mathrm{V}=2 / 3 \mathrm{ab}^{2}$, where $\mathrm{a}$ is the longest diameter and $\mathrm{b}$ is the shortest diameter. The survival times of the nude mice were recorded. Once the animals died, the tumor tissues were removed immediately for additional analysis.

Histological analysis. The formalin-fixed mouse tissues were embedded in paraffin. Serial 5- $\mu \mathrm{m}$ sections were obtained with a Leica microtome. The sections were then dewaxed, hydrated and incubated in methanol- $\mathrm{H}_{2} \mathrm{O}_{2}$ for 20 min to remove endogenous peroxidase. The slides of the tumor tissues were incubated sequentially with rabbit monoclonal antibody to human $\beta \mathrm{G}$, horseradish peroxidase-conjugated goat anti-rabbit IgG. The sections were counterstained with hematoxylin and evaluated independently in a blinded manner.

Statistical analysis. Statistical analysis was performed using the SPSS 11.5 software package for Windows (SPSS, Chicago, IL, USA). Statistical significance was assessed by comparing mean \pm SD values with the Student's t-test for independent groups. Tumor volumes were analyzed by the analysis of covariance (ANCOVA) method followed by LSD as post-hoc, with comparisons between treatment groups made by covariance test with the beginning differences occurring by grouping eliminated. $\mathrm{P}<0.05$ was considered to indicate a statistically significant difference.

\section{Results}

Targeted gene expression is amplified using the PSA promoter-controlled GAL4-regulated bicistronic adenovirus. To construct the recombinant adenovirus, the human PSA promoter gene was amplified from the PSA-producing prostate cancer cell line LNCaP, and the human $\beta \mathrm{G}$ gene was amplified from the human hepatocarcinoma cell line HepG2. New bicistronic shuttle plasmids (pshuttle-PSAP-GV16- $\beta$ G, pshuttle-PSAP-GV16-luc and pshuttle-PSAP-GV16-EGFP) were constructed by replacing the hTERT promoter and TRAIL gene in pshuttle-hTERT-GV16-TRAIL (15) with the PSA promoter and the $\beta \mathrm{G}$, luciferase or EGFP gene, respectively. Recombinant vectors (Ad/PSAP-GV16- $\beta$ G, Ad/PSAP-GV16-luc and Ad/PSAP-GV16-EGFP) were generated by homologous recombination. By using these adenoviruses, the expression of $\beta G$, luciferase or EGFP was controlled by the PSA promoter and the GAL4-regulatory system (Fig. 1A). The shuttle plasmids in which $\beta G$, luciferase or EGFP expression was controlled directly by the PSA promoter were also constructed (pshuttle-PSAP- $\beta \mathrm{G}$, pshuttle-PSAP-luc and pshuttle-PSAP-EGFP) and recombinant vectors (Ad/PSAP- $\beta$ G, Ad/PSAP-Luc and Ad/PSAP-EGFP) were generated by homologous recombination.

To evaluate the effectiveness of the augmented expression system, PSA-producing LNCaP cells and PSA-non-producing DU145 cells were infected with the control adenovirus, Ad/PSAP-GV16-Luc or Ad/PSAP-Luc at a multiplicity of infection (MOI) of 1,000 viral particles/cell. The cells were lysed and luciferase activities were determined $48 \mathrm{~h}$ after the infection. In the PSA-producing LNCaP cells, there was a relatively low luciferase level after infection with Ad/PSAP-Luc, while the luciferase activity increased 80 - to 100-fold in the Ad/PSAP-GV16-Luc-infected cell lysates $(\mathrm{P}=0.001)$. In contrast, luciferase activity was scarcely detected in the PSA-non-producing DU145 cells after infection with either Ad/PSAP-Luc or Ad/PSAP-GV16-Luc (Fig. 1B).

Furthermore, when the LNCaP and DU145 cells were infected with the control adenovirus, Ad/PSAP-GV16-EGFP or Ad/PSAP-EGFP for $48 \mathrm{~h}$, the expression of EGFP was only observed in the PSA-producing LNCaP cells, with Ad/PSAP-GV16-EGFP-infected cells showing significantly stronger EGFP expression than the Ad/PSAP-EGFP-infected cells (Fig. 1C). EGFP expression was hardly observed in the PSA-non-producing DU145 cells either infected with Ad/PSAP-EGFP or Ad/PSAP-GV16-EGFP (data not shown).

Amplified $\beta G$ expression significantly increases the sensitivity to DOX-GA3 and the bystander effect in LNCaP cells. Expression of $\beta \mathrm{G}$ was detected $48 \mathrm{~h}$ after the LNCaP and DU145 cells were infected with the control adenovirus, Ad/PSAP- $\beta$ G or Ad/PSAP-GV16- $\beta$ G at an MOI of 1,000 viral particles/cell. The results of RT-PCR and western blot analysis showed that the $\beta \mathrm{G}$ expression was only detected in the $\mathrm{LNCaP}$ cells, and $\beta \mathrm{G}$ expression was significantly amplified in the Ad/PSAP-GV16- $\beta$ G-infected cells compared with the Ad/PSAP- $\beta$ G-infected cells (Fig. $2 \mathrm{~A}$ and B).

The cytotoxicity of DOX-GA3 on the infected LNCaP and DU145 cells was evaluated by MTT assay. When different amounts of DOX-GA3 were added into the supernatant of 


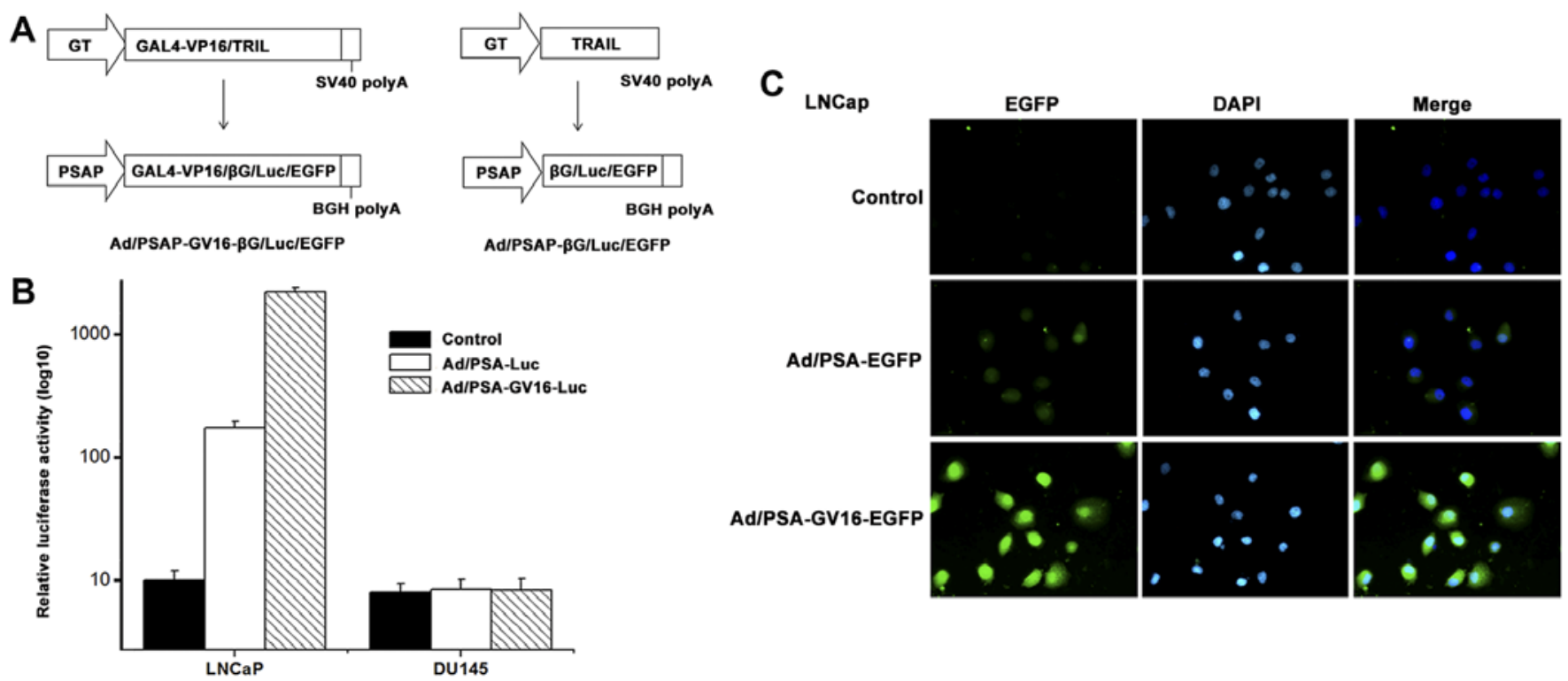

Figure 1. Targeted gene expression is amplified by the GAL4-regulated bicistronic adenovirus controlled by the PSA promoter. (A) Schematic diagram of recombinant bicistronic adenovirus vectors. (B) Luciferase activity detected in LNCaP and DU145 cell lysates after they were infected with control adenovirus, Ad/PSAP-GV16-luc or Ad/PSAP-luc for $48 \mathrm{~h}$. (C) Fluorescence observation of LNCaP cells after infection with the control adenovirus, Ad/PSAP-GV16-EGFP or Ad/PSAP-EGFP for $48 \mathrm{~h}$. Nuclei were counterstained with 4',6-diamidino-2-phenylindole (DAPI). Magnification, $\mathrm{x} 400$. Data are shown as mean $\pm \mathrm{SD}$. ${ }^{* * *} \mathrm{P}<0.001$ vs. the Ad/PSAP-Luc group.

the cells which were infected for $48 \mathrm{~h}$, both Ad/PSAP- $\beta \mathrm{G}$ and Ad/PSAP-GV16- $\beta$ G-infected LNCaP cells showed an apparent dose-dependent relationship between DOX-GA3 concentration and growth inhibition (Fig. 2C). DOX-GA3 cytotoxity on the Ad/PSAP-GV16- $\beta$ G-infected cells was much more potent than on the Ad/PSAP- $\beta$ G-infected group, indicating an increased sensitivity to DOX-GA3 $(\mathrm{P}=0.01)$. In the DU145 cells, the DOX-GA3 cytotoxity was comparable whether they were infected with the control adenovirus or the $\beta$ G-expressing adenoviruses (Fig. 2D).

To detect the time-dependent cytotoxity of DOX-GA3 on LNCaP and DU145 cells after they were infected with Ad/PSAP- $\beta$ G or Ad/PSAP-GV16- $\beta \mathrm{G}, 10 \mu \mathrm{g} / \mathrm{ml}$ DOX-GA3 was added into the supernatant of the infected cells for different times before MTT assay. As shown in Fig. 2E, obvious cytotoxity was observed in both the Ad/PSAP- $\beta$ G- and Ad/PSAP-GV16- $\beta$ G-infected LNCaP cells, and the cytotoxity was more significant in the Ad/PSAP-GV16- $\beta$ G-infected cells $(P=0.027)$, indicating the increased sensitivity to DOX-GA3 in these cells. On the other hand, DU145 cells did not show increased sensitivity to DOX-GA3 whether they were infected with Ad/PSAP- $\beta$ G or Ad/PSAP-GV16- $\beta$ G (Fig. 2F).

Next, to evaluate the effect of the amplified $\beta \mathrm{G}$ expression on the bystander effect, different percentages of infected LNCaP cells were mixed with uninfected cells before DOX-GA3 $(10 \mu \mathrm{g} / \mathrm{ml})$ was applied and incubated for $72 \mathrm{~h}$. In the Ad/PSAP-GV16- $\beta$ G-infected group, the cells were almost completely killed after the DOX-GA3 incubation with only $\sim 40 \%$ infected cells. Even when only $20 \%$ of the Ad/PSA-GV16- $\beta$ G-infected cells were applied, $\sim 80 \%$ of total cells were killed, showing a stronger bystander effect than that noted in the Ad/PSA- $\beta$ G-infected cells $(\mathrm{P}=0.001)$ (Fig. $2 \mathrm{G})$.
Amplified and selective target gene expression in vivo using the GAL4-regulated bicistronic adenovirus. To evaluate whether selective and amplified expression of target genes can be achieved in vivo, nude mouse models with PSA-producing LNCaP xenografts were established. When tumors reached a diameter of $\sim 0.3-0.5 \mathrm{~cm}$, control adenovirus, Ad/PSAP-GV16-Luc or Ad/PSAP-Luc were injected into the tail vein, mice were sacrificed $48 \mathrm{~h}$ later, and luciferase activities were determined in the tumors and different tissues. Results showed that the luciferase activity was only detected in tumor lysates of both the Ad/PSAP-Luc- and Ad/PSAP-GV16-Luc-infected mice, and the luciferase activity was significantly higher in the Ad/PSAP-GV16-Luc-infected mice than that in the Ad/PSAP-Luc-infected mice ( $\mathrm{P}=0.001)$ (Fig. 3A).

To directly observe the selective and amplified gene expression, mice with LNCaP xenografts were infected with the control adenovirus, Ad/PSAP-GV16-EGFP or Ad/PSAP-EGFP for $48 \mathrm{~h}$ before tumors and different tissues were snap-frozen and cryosectioned for direct fluorescence observation. EGFP expression was only observed in the tumors and significantly stronger fluorescence was observed in the Ad/PSAP-GV16-EGFP-infected mice than that in the Ad/PSAP-EGFP-infected mice (Fig. 3B).

Targeted and amplified $\beta G$ expression combined with DOX-GA3 administration effectively suppresses the growth of PSA-producing tumors. To confirm the targeted and amplified $\beta \mathrm{G}$ expression in vivo, the control adenovirus, Ad/PSAP- $\beta \mathrm{G}$ or Ad/PSAP-GV16- $\beta$ G were intravenously injected into nude mice with LNCaP xenografts. The mice were sacrificed $48 \mathrm{~h}$ after the injection, and tumors were lysed to detected $\beta \mathrm{G}$ expression by dot blot and western blot analysis. $\beta \mathrm{G}$ expression was detected 


\section{A}

Control Ad/PSA- $\beta$ G Ad/PSA-GV16- $\beta$ G

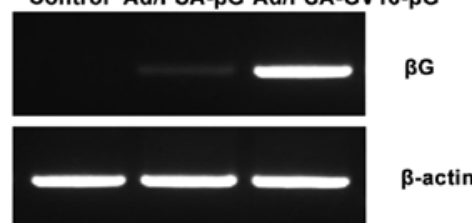

B Control Ad/PSA- $\beta$ G Ad/PSA-GV16- $\beta$ G
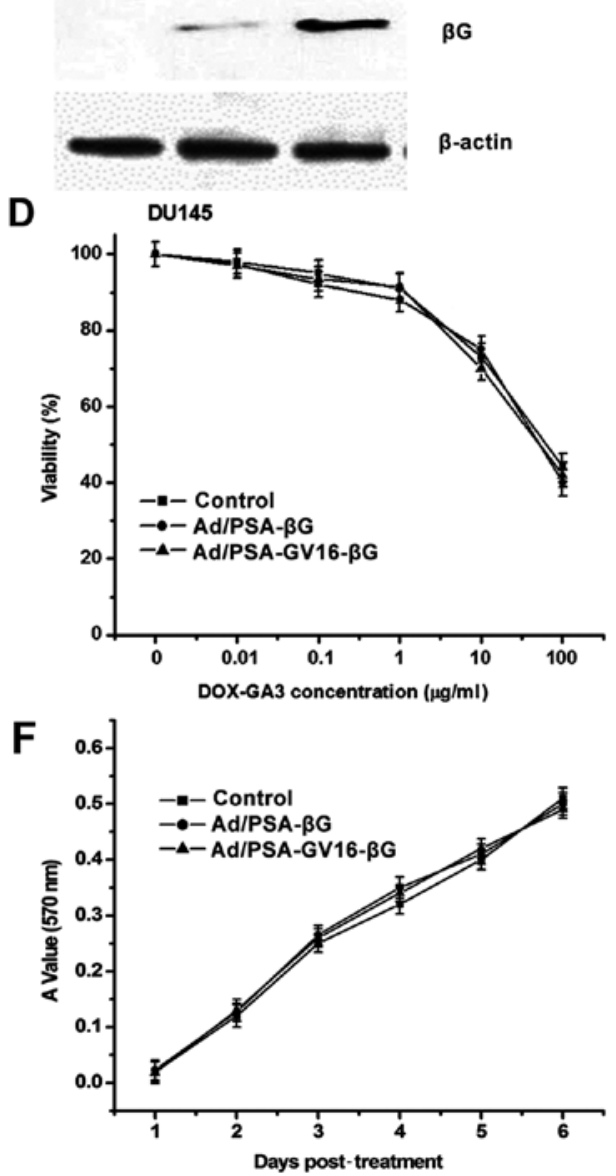

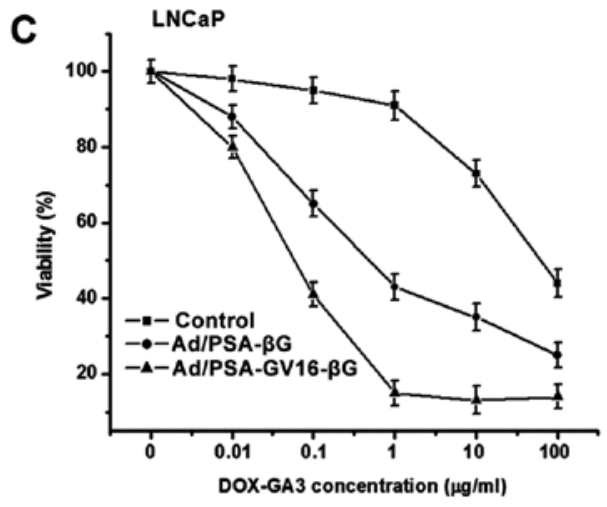

E
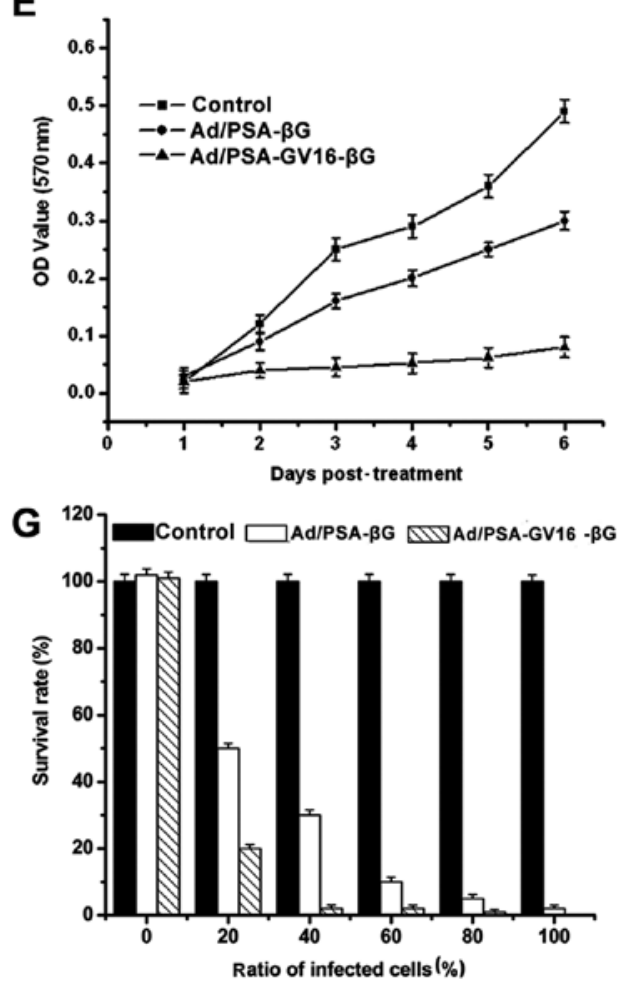

Figure 2. Amplified $\beta$ G expression increases the sensitivity to DOX-GA3 and the bystander effect in LNCaP cells. LNCaP cells were infected with the control adenovirus, Ad/PSAP-GV16- $\beta$ G or Ad/PSAP- $\beta$ G for $48 \mathrm{~h}$ before they were lysed, and the $\beta \mathrm{G}$ expression at the mRNA level by RT-PCR (A) and protein level by western blot analysis (B) was evaluated. (C-F) The dose-dependent and time-dependent cytotoxicity of DOX-GA3 on LNCaP and DU145 cells was evaluated by MTT assays after they were infected with the control adenovirus, Ad/PSAP- $\beta$ G or Ad/PSAP-GV16- $\beta$ G for 48 h and incubated with DOX-GA3 at the indicated doses for the indicated times. $(\mathrm{G})$ The bystander effect in infected LNCaP cells was detected after they were incubated with different ratios of uninfected cells. Data are shown as mean $\pm \mathrm{SD} .{ }^{*} \mathrm{P}<0.05,{ }^{* *} \mathrm{P}<0.01,{ }^{* * * *} \mathrm{P}<0.001$ vs. the Ad/PSAP- $\beta \mathrm{G}$ group.
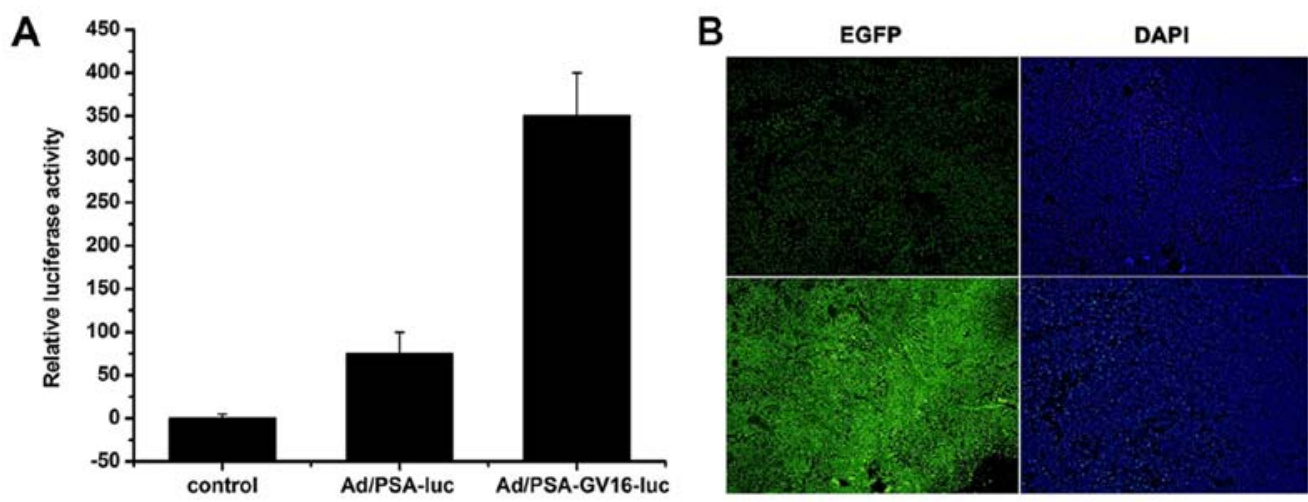

Ad/ PSA-EGFP

Ad/PSA-GV16-EGFP

Figure 3. Amplified transgene expression is realized in vivo by using the GAL4-regulated bicistronic adenovirus. Nude mice with PSA-producing LNCaP xenografts were established. (A) Luciferase activities were determined in tumors and different tissues $48 \mathrm{~h}$ after the injection of the control adenovirus, Ad/PSAP-GV16-Luc or Ad/PSAP-Luc. (B) Fluorescence observation in tumors and different tissues $48 \mathrm{~h}$ after the injection of the control adenovirus, Ad/PSAP-GV16-EGFP or Ad/PSAP-EGFP. Magnification, x200. Data are shown as mean \pm SD. ${ }^{* * *}$ P<0.001 vs. the Ad/PSAP-Luc group. 


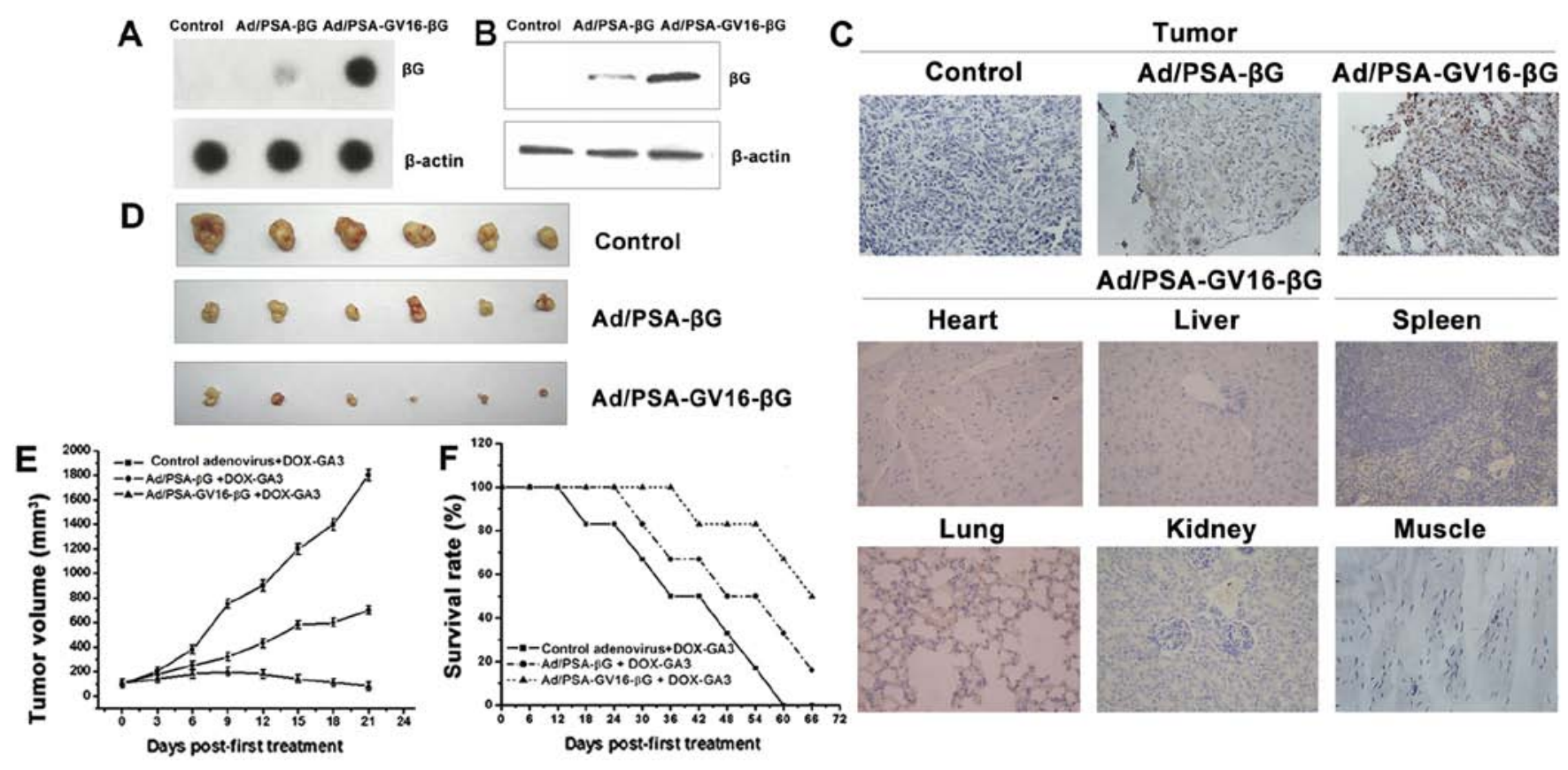

Figure 4. Targeted and amplified $\beta \mathrm{G}$ expression plus DOX-GA3 administration effectively suppresses tumor growth. Control adenovirus, Ad/PSAP- $\beta \mathrm{G}$ or Ad/PSAP-GV16- $\beta$ G were intravenously injected into nude mice with PSA-producing LNCaP xenografts. $\beta$ G expression was detected $48 \mathrm{~h}$ after the injection at the mRNA level by dot blot (A) and protein level by western blot analysis (B). (C) Selective and increased $\beta$ G expression was confirmed by immunohistochemical staining. $\beta G$ expression was undetectable in the heart, liver, spleen, lung, kidney, or muscle. (D) After being inoculated after 6 weeks, the tumors were extracted from nude mice treated with the control adenovirus, Ad/PSAP- $\beta$ G or Ad/PSAP-GV16- $\beta$ G infection and DOX-GA3. Tumor volume (E) and survival rate $(F)$ in each group were calculated every three or six days after mice were treated with the control adenovirus, Ad/PSAP- $\beta$ G or Ad/PSAP-GV16- $\beta$ G infection and DOX-GA3. Data are shown as mean $\pm \mathrm{SD}$. ${ }^{*} \mathrm{P}<0.05$ vs. the Ad/PSAP- $\beta \mathrm{G}$ group.

in the Ad/PSAP- $\beta$ G- and Ad/PSAP-GV16- $\beta$ G-infected groups, with the Ad/PSAP-GV16- $\beta$ G-infected mice showing significantly higher $\beta \mathrm{G}$ expression than the Ad/PSAP- $\beta$ G-infected mice (Fig. 4A and B). Results of the immunohistochemical staining confirmed the increased expression of $\beta G$, and there was no detectable $\beta \mathrm{G}$ expression in the heart, liver, spleen, lung, kidney, or muscle, indicating the selective $\beta G$ expression in PSA-producing tumor tissue (Fig. 4C).

To evaluate the therapeutic effect of this strategy, $500 \mathrm{mg} / \mathrm{kg}$ DOX-GA3 was simultaneously injected with the control adenovirus, Ad/PSAP- $\beta$ G or Ad/PSAP-GV16- $\beta$ G into the established nude mouse model. After twice weekly sequential intravenous injections of adenovirus and DOX-GA3, tumor volume was monitored every 3 days. Treatment with Ad/PSAP-GV16- $\beta$ G and $500 \mathrm{mg} / \mathrm{kg}$ DOX-GA3 resulted in more effective tumor growth inhibition than the Ad/PSAP- $\beta \mathrm{G}$ and DOX-GA3-treated group $(\mathrm{P}<0.05)$ (Fig. 4D and E). Survival time of the treated mice was also evaluated. In mice treated with Ad/PSAP- $\beta$ G or Ad/PSAP-GV16- $\beta$ G and DOX-GA3, the survival time was prolonged, especially in the Ad/PSAP-GV16- $\beta$ G-infected group (Fig. 4 F), and the difference was significant $(\mathrm{P}<0.05)$.

\section{Discussion}

The aim of the present study was to investigate whether a GAL4-regulated bicistronic adenovirus could achieve selective and amplified $\beta G$ expression in PSA-producing prostate cancer cells by using the PSA promoter. If successful, this suicide gene system would have potential for application in the treatment of prostate cancer. Our results showed that by using the system, selective and amplified expression of both reporter genes and $\beta \mathrm{G}$ were achieved in the PSA-producing LNCaP cells but not in the PSA-non-producing DU145 cells. This selective and amplified $\beta G$ expression combined with the prodrug DOX-GA3 was shown to have strong cytotoxicity and a strong bystander effect in the PSA-producing LNCaP cells, but not in the PSA-non-producing DU145 cells.

Because of the unique bystander effect, suicide gene therapy is considered to be one of the most effective strategies for cancer therapy (18-22). Compared with other organs, the prostate is more suitable for suicide gene therapy for the following reasons. i) It is easy to access by transurethral or transrectal route; ii) several proteins are known to be expressed in a tissue-specific manner, including prostate-specific antigen (PSA), human glandular kallikrein 2 (hKLK2) and prostate-specific membrane antigen (PSMA), showing great potential for tissue-specific targeted expression of toxic gene by using tissue-specific promoter and (iii) both normal and malignant cells in the prostate have been shown to have abundant connexin proteins. The connexin proteins assemble to form tiny water-filled channels to execute gap junction functions, which play a significant role in the bystander effect $(23,24)$.

There are two main approaches to achieve high and specific expression of a suicide gene for specific killing. One is antibody-directed enzyme prodrug therapy (ADEPT), in which a suicide gene protein is fused with a tumor-specific antibody which can specifically direct the suicide gene protein to the tumor $(6,11,25-27)$. Another promising approach is 
gene-directed enzyme prodrug therapy (GDEPT) in which the expression of a suicide gene is controlled by a tumor-specific promoter which can mediate its expression specifically in tumors (5,28-30). Compared with the ADEPT strategy, GDEPT seems to be more attractive since it can avoid the instability and the fast renal clearance of the antibody-suicide gene fusion protein. However, although specific suicide gene expression can be achieved using tumor-specific promoters such as the carcinoembryonic antigen (CEA) promoter, PSA promoter and prostate-specific membrane antigen (PSMA) promoter (31-35), the relatively weak transcription activity of these promoters often results in poor expression of suicide genes which severely attenuates the therapeutic effect. To overcome this problem, several augmented expression systems have been designed to improve the targeted expression of therapeutic genes, among which the GAL4-VP16 regulation system seemed to be most attractive (15,36-38). In this system, the target gene expression is controlled by a synthesized GAL4/TATA (GT) promoter, which can be transactivated by GAL4-VP16, whose expression is controlled by a tissue-specific promoter. The GAL4-VP16 regulatory system is also attractive for the following reasons. i) GAL4/TATA (GT) promoter has extremely low basal activity in most organs and ii) the GT promoter is highly inducible when co-delivered with the transactivating protein GAL4-VP16 at a low dose.

In the present study, we first evaluated the effectiveness of the GAL4 regulatory system by using luciferase and EGFP as reporter genes. By using the PSA promoter-controlled and GAL4-VP16-regulated bicistronic adenovirus, we showed that expression of the reporter gene could be augmented 80 - to 100-fold, while the specificity was well maintained. Amplification of $\beta \mathrm{G}$ expression was also achieved by using this expression system, and because of the selective and amplified $\beta G$ expression in PSA-producing LNCaP cells, their sensitivity to DOX-GA3 was significantly improved, and a stronger bystander effect was also observed in these cells. Our in vivo results showed that the specific and augmented $\beta \mathrm{G}$ expression together with the application of its prodrug DOX-GA3 resulted in significantly stronger suppression on tumor growth in nude mice with PSA-producing LNCaP xenografts.

$\beta \mathrm{G}$ is an acid hydrolase present in many human tissues. It can convert the nontoxic glucuronide prodrug with glycoside bonds into toxic glucuronic acid. Normally the endogenous $\beta G$ expression is very low, but under certain conditions, for example, in several types of tumor tissue, the expression of $\beta G$ may increase. To test whether the relatively low $\beta \mathrm{G}$ expression may result in obvious toxicity after the application of its prodrug DOX-GA3, we detected the ALT and AST levels in the adenovirus-infected and DOX-GA3-treated mice, and the results did not show an obvious increase in ALT and AST levels (data not shown). Our immunohistochemical staining of different tissues also did not show any obvious changes. Potential toxicity of this system still needs to be evaluated before clinical use.

In conclusion, this is the first trial that demonstrates that selective and amplified suicide gene expression can be achieved by using a GAL4-regulated bicistronic adenovirus controlled by the PSA promoter, and that the selective and amplified $\beta G$ expression can strongly inhibit the growth of prostate cancer growth. The combination of the GAL4 system and the PSA promoter resulted in high gene expression efficiency while maintaining good specificity. These results support the possibility of the application of the PSA promoter-controlled $\beta G$ suicide gene system for prostate cancer gene therapy.

\section{Acknowledgements}

The present study was supported by grants from the Nature Science Foundation of China (project nos. 81372741, 81172146, 81372771, 81171924 and 30300413).

\section{References}

1. MacRae EJ, Giannoudis A, Ryan R, Brown NJ, Hamdy FC, Maitland N and Lewis CE: Gene therapy for prostate cancer: Current strategies and new cell-based approaches. Prostate 66: 470-494, 2006.

2. Trojan L, Kiknavelidze K, Knoll T, Alken P and Michel MS: Prostate cancer therapy: Standard management, new options and experimental approaches. Anticancer Res 25: 551-561, 2005.

3. Ward JF and Moul JW: Biochemical recurrence after definitive prostate cancer therapy. Part I: Defining and localizing biochemical recurrence of prostate cancer. Curr Opin Urol 15: 181-186, 2005.

4. Ward JF and Moul JW: Biochemical recurrence after definitive prostate cancer therapy. Part II: Treatment strategies for biochemical recurrence of prostate cancer. Curr Opin Urol 15: 187-195, 2005.

5. de Graaf M, Boven E, Scheeren HW, Haisma HJ and Pinedo HM: Beta-glucuronidase-mediated drug release. Curr Pharm Des 8: 1391-1403, 2002.

6. Houba PH, Boven E, van der Meulen-Muileman IH, Leenders RG, Scheeren JW, Pinedo HM and Haisma HJ: Pronounced antitumor efficacy of doxorubicin when given as the prodrug DOX-GA3 in combination with a monoclonal antibody beta-glucuronidase conjugate. Int J Cancer 91: 550-554, 2001.

7. Houba PH, Boven E, van der Meulen-Muileman IH, Leenders RG, Scheeren JW, Pinedo HM and Haisma HJ: A novel doxorubicin-glucuronide prodrug DOX-GA3 for tumour-selective chemotherapy: Distribution and efficacy in experimental human ovarian cancer. Br J Cancer 84: 550-557, 2001.

8. van Dillen IJ, Mulder NH, Vaalburg W, de Vries EF and Hospers GA: Influence of the bystander effect on HSV-tk/GCV gene therapy. A review. Curr Gene Ther 2: 307-322, 2002

9. Mesnil M and Yamasaki H: Bystander effect in herpes simplex virus-thymidine kinase/ganciclovir cancer gene therapy: Role of gap-junctional intercellular communication. Cancer Res 60: 3989-3999, 2000.

10. Fonseca MJ, Storm G, Hennink WE, Gerritsen WR and Haisma J: Cationic polymeric gene delivery of beta-glucuronidase for doxorubicin prodrug therapy. J Gene Med 1: 407-414, 1999.

11. de Graaf M, BovenE, Oosterhoff D, van der Meulen-Muileman IH, Huls GA, Gerritsen WR, Haisma HJ and Pinedo HM: A fully human anti-Ep-CAM scFv-beta-glucuronidase fusion protein for selective chemotherapy with a glucuronide prodrug. Br J Cancer 86: 811-818, 2002.

12. Wei G, Loktionova NA, Pegg AE and Moschel RC: Beta-glucuronidase-cleavable prodrugs of $\mathrm{O}^{6}$-benzylguanine and O'-benzyl-2'-deoxyguanosine. J Med Chem 48: 256-261, 2005.

13. Houba PH, Boven E, Erkelens CA, Leenders RG, Scheeren JW, Pinedo HM and Haisma HJ: The efficacy of the anthracycline prodrug daunorubicin-GA3 in human ovarian cancer xenografts. Br J Cancer 78: 1600-1606, 1998.

14. Sperker B, Werner U, Mürdter TE, Tekkaya C, Fritz P, Wacke R, Adam U, Gerken M, Drewelow B and Kroemer HK: Expression and function of beta-glucuronidase in pancreatic cancer: Potential role in drug targeting. Naunyn Schmiedebergs Arch Pharmacol 362: 110-115, 2000.

15. Park HS, Cheon J, Cho HY, Ko YH, Bae JH, Moon DG and Kim JJ: In vivo characterization of a prostate-specific antigen promoter-based suicide gene therapy for the treatment of benign prostatic hyperplasia. Gene Ther 10: 1129-1134, 2003.

16. Yu D, Chen D, Chiu C, Razmazma B, Chow YH and Pang S: Prostate-specific targeting using PSA promoter-based lentiviral vectors. Cancer Gene Ther 8: 628-635, 2001. 
17. Lin T, Gu J, Zhang L, Huang X, Stephens LC, Curley SA and Fang B: Targeted expression of green fluorescent protein/tumor necrosis factor-related apoptosis-inducing ligand fusion protein from human telomerase reverse transcriptase promoter elicits antitumor activity without toxic effects on primary human hepatocytes. Cancer Res 62: 3620-3625, 2002.

18. Nasu Y, Kusaka N, Saika T, Tsushima T and Kumon H: Suicide gene therapy for urogenital cancer: Current outcome and prospects. Mol Urol 4: 67-71, 2000.

19. Yazawa K, Fisher WE and Brunicardi FC: Current progress in suicide gene therapy for cancer. World J Surg 26: 783-789, 2002.

20. Poulsen TT, Pedersen N and Poulsen HS: Replacement and suicide gene therapy for targeted treatment of lung cancer. Clin Lung Cancer 6: 227-236, 2005.

21. Nicholas TW, Read SB, Burrows FJ and Kruse CA: Suicide gene therapy with Herpes simplex virus thymidine kinase and ganciclovir is enhanced with connexins to improve gap junctions and bystander effects. Histol Histopathol 18: 495-507, 2003.

22. Fillat C, Carrió M, Cascante A and Sangro B: Suicide gene therapy mediated by the herpes simplex virus thymidine kinase gene/ganciclovir system: Fifteen years of application. Curr Gene Ther 3: 13-26, 2003.

23. Satoh T, Irie A, Egawa S and Baba S: In situ gene therapy for prostate cancer. Curr Gene Ther 5: 111-119, 2005.

24. Shalev M, Kadmon D, Teh BS, Butler EB, Aguilar-Cordova E, Thompson TC, Herman JR, Adler HL, Scardino PT and Miles BJ: Suicide gene therapy toxicity after multiple and repeat injections in patients with localized prostate cancer. J Urol 163 $1747-1750,2000$

25. Biela BH, Khawli LA, Hu P and Epstein AL: Chimeric TNT-3/ human beta-glucuronidase fusion proteins for antibody-directed enzyme prodrug therapy (ADEPT). Cancer Biother Radiopharm 18: 339-353, 2003.

26. Haisma HJ, Sernee MF, Hooijberg E, Brakenhoff RH, vd Meulen-Muileman IH, Pinedo HM and Boven E: Construction and characterization of a fusion protein of single-chain anti-CD20 antibody and human beta-glucuronidase for antibody-directed enzyme prodrug therapy. Blood 92: 184-190, 1998.

27. Kaliberov SA, Chiz S, Kaliberova LN, Krendelchtchikova V, Della Manna D, Zhou T and Buchsbaum DJ: Combination of cytosine deaminase suicide gene expression with DR5 antibody treatment increases cancer cell cytotoxicity. Cancer Gene Ther 13: 203-214, 2006.

28. Djeha HA, Todryk SM, Pelech S, Wrighton CJ, Irvine AS, Mountain A and Lipinski KS: Antitumor immune responses mediated by adenoviral GDEPT using nitroreductase/CB1954 is enhanced by high-level coexpression of heat shock protein 70 Cancer Gene Ther 12: 560-571, 2005.
29. Miki K, Xu M, Gupta A, Ba Y, Tan Y, Al-Refaie W, Bouvet M, Makuuchi M, Moossa AR and Hoffman RM: Methioninase cancer gene therapy with selenomethionine as suicide prodrug substrate. Cancer Res 61: 6805-6810, 2001.

30. Denny WA: Prodrugs for gene-directed enzyme-prodrug therapy (suicide gene therapy). J Biomed Biotechnol 2003: 48-70, 2003.

31. Dabrowska A, Szary J, Kowalczuk M, Szala S and Ugorski M: CEA-negative glioblastoma and melanoma cells are sensitive to cytosine deaminase/5-fluorocytosine therapy directed by the carcinoembryonic antigen promoter. Acta Biochim Pol 51: $723-732,2004$

32. Ikegami S, Tadakuma T, Suzuki S, Yoshimura I, Asano T and Hayakawa $\mathrm{M}$ : Development of gene therapy using prostate-specific membrane antigen promoter/enhancer with Cre Recombinase/LoxP system for prostate cancer cells under androgen ablation condition. Jpn J Cancer Res 93: 1154-1163, 2002.

33. Peng W, Chen J, Huang YH and Sawicki JA: Tightly-regulated suicide gene expression kills PSA-expressing prostate tumor cells. Gene Ther 12: 1573-1580, 2005.

34. Qiao J, Doubrovin M, Sauter BV, Huang Y, Guo ZS, Balatoni J, Akhurst T, Blasberg RG, Tjuvajev JG, Chen SH, et al: Tumor-specific transcriptional targeting of suicide gene therapy. Gene Ther 9: 168-175, 2002.

35. Ueda K, Iwahashi M, Nakamori M, Nakamura M, Matsuura I, Yamaue $\mathrm{H}$ and Tanimura $\mathrm{H}$ : Carcinoembryonic antigen-specific suicide gene therapy of cytosine deaminase/5-fluorocytosine enhanced by the cre/loxP system in the orthotopic gastric carcinoma model. Cancer Res 61: 6158-6162, 2001.

36. Koch PE, Guo ZS, Kagawa S, Gu J, Roth JA and Fang B: Augmenting transgene expression from carcinoembryonic antigen (CEA) promoter via a GAL4 gene regulatory system. Mol Ther 3: 278-283, 2001.

37. Ray S, Paulmurugan R, Hildebrandt I, Iyer M, Wu L, Carey M and Gambhir SS: Novel bidirectional vector strategy for amplification of therapeutic and reporter gene expression. Hum Gene Ther 15: 681-690, 2004.

38. Iyer M, Salazar FB, Lewis X, Zhang L, Wu L, Carey M and Gambhir SS: Non-invasive imaging of a transgenic mouse model using a prostate-specific two-step transcriptional amplification strategy. Transgenic Res 14: 47-55, 2005. 